\title{
Ueber die chemische Constitution der salicylsauren Salze.
}

Nach der bisherigen Ansicht bietet die Salicylsiiure die eigenthümliche Anomalie dar, obwohl einbasisch, mit dem Acthyl, Methyl- und Amylalkohol saure Verbindungen zu bilden, welche gleiche Süttigungscapacität besitzen, als die freie Säure. Wenig befriedigt von Gerhardt's Erklïrung dieses Verhaltens (Annal. der Chemie u. Pharm. Bd. 89. S. 360), unternahm Piria eine neue Untersuchung der salicylsauren Salze, welche ergab, dass die nach den Arbeiten von Gahours und Gerhardt als einbasisch betrachtete Salicylsiure zu ren zweibasischen säuren zu zïhlen ist.

I) Salicylsäure, deren sich Piria zur Darstellung der unten beschriebenen neu entdeckten Salze bediente, wurde erhalten, indem er die im Handel vorkommende Essenz des winter-grete.1. nit kqustischer Kalilösung kochte und das Product durch salzsiiure zersetzte. Die von ihm dargestellten Salze mit 2 Aeq. Basis sind die des Baryts, Kalks, Bleies, Kupters und dic Doppelsalze des Kupfers und Baryts, des Kupfers und Kalis. Mit Ausnahme des letzten sind alle andern wenig löslich in Wasser, völlig unlöslich in Alkohol und Aether. Der grössere Theil derselben hat dentlich alkalische Reactionen und wird theilweise durch freie Kohlensüure zersetzt, besonders im feuchten Zustande. Unter Anwendung der von den Chemikern angenommenen Grundsätze für die Nomenclatur der Salze anderer Familien, wie der kohlensauren, borsauren u. s. w., nennt P'iria, ohne Rücksicht auf die Finwirkung, welche sie anf die Farbstoffe änssern, Bisalicylate oder saure salicylsaure Salze jene, welche nur 1 Aeq. Basis enthalten und bis jetzt unter dem Namen der Salicylate bekannt waren, indem er die lotztere Benennung den neu von ihm entdeckten Salzen mit 2 Aeq. Basis vindicirt.

Salicylsaurer Baryt. Dieses Salz wird leicht erhalten, indem man das saure Salz durch einen Ueberschuss der Basis niederschlägt. Zu dem kinde bereitet man sauren salicylsauren Baryt nach der Vorschrift von Cahours, indem man Salicylsäure mit kohlensaurem Baryt sieden lïsst und die siedenheisse Lösung filtrirt; darauf giesst man zu der filtrirten Fliissigkeit eine concentrirte, heisse Lösung von Barythydrat; das neutrale Salz, wel- 
ches sehr wenig löslich ist, setzt sich in Form selir weisser krystallinischer Schuppen ab. Tm dasselbe zu reinigen, muss man es zum zweiten Male aus der heissen wässerigen I.ösung herauskrystallisiren lassen; da es aber wenig löslich ist, und seine Lösung dureh Kohlensäure zersetzt wird, so muss man es in Berührung mit einer grossen Menge vorler zum Kochen gobrachten Wassers erhitzen, die lü̈sung sehr schnell filtriren, indem man sie so viel als möglich vor dem Zutritte der Luft bewnhrt, und in einer Flasche auffangen, welche sich luftlicht versehliessen lïsst. Nach Verlauf' von ein oder zwei 'Tagen kann man die Krystalle aus der Mlutterlauge herausnehmen und zwischen Löschpapier unter ciner Glocke trocknen, welche gebrannten Kalk enthält. Das Sal\% bildet weisse, undurchsichtige, kleine Nadeln, welche um cinen gemeinsamen Mittelpunct gruppirt sind. Die Lösung bliut das rothe Lackmuspapier stark und wird durch Kohlensiuure zersetzt, welche die Hälfte der Basis als kohlensaures Salz nicderschlägt, wobei das neutrale Salz in das saure übergeht.

Der salicylsaure Baryt verliert beim Erwärmen 4 Aeq. Wasser. 2,275 Grmn. der Krystalle verloren, in einem durch Kalilauge von F'euchtigkeit und Kohlensïure befreiten Luftstrome auf $140^{\circ}$ erwärmt, $0,261 \mathrm{Grm} .=11,47$ Procent. Indem die Temperatur des Luftbades auf $160^{\circ}$ gesteigert wurde, erlitt die Substanz keine weitere (icwichtsverminderung. 1,535 (irm. des neutralen Salzes verloren bei $160^{0} 0,1735=11,30$ Proc. Krystallisationswasser, während die Rechnung 11,63 Proc. erfordert.

Das Baryum wurde aus der Menge des schwefelsauren Baryts bestimmt, welche als Rückstand beim Glühen des Salzes in Berührung mit Schwefelsäure blieb. 0,409 salicylsaurer Baryt hinterliessen 0,309 schwefelsauren 13aryt. 0,574 des Salzes gaben 0,434 schwefelsauren Baryt.

Uim den Wasserstoff und Kohlenstoff zu bestimmen, wurde die Untersuchung auf dic gewöhnliche Art ausgeführt, indem man die Vorsicht gebrauchte, die Verbrennung in einem Sauerstoffatrome zu vollenden; da aber ein Theil der Kollensäure vom Baryt zurückgehalten wird, so nahm P'iria an, dass von jedem Aequivalente des Salzes, welches 2 Aeq. Basis auf 14 Aeq. Kohlenstoff enthält, während der Verbrennung $6 / 7$ des ganzen Kohlenstoffgehalts sich in Form von Kohlensiiure verflüchtigen, und dass $1 / 7$ in der Röhre als kohlensaurer Baryt zuriickbleibt. Da nun die theoretische Zusammensetzung nach 
der Formel $\mathrm{C}^{14} \mathrm{H}^{4} \mathrm{Ba}^{2} \mathrm{O}^{6}+4 \mathrm{Ag}$ berechnet, 27,13 Proc. Kohlenstoff geben würde, so hat er der durch die Analyse erbaltenen Menge des Kohlenstoffs $\frac{\mathbf{2 7 , 1 3}}{\mathbf{7}}=3,88$ hinzugefügt, welche aus den oben angeführten Ursachen in Verbindung mit Baryt zurückgeblieben sein müssen. Hier folgen übrigens die unmittelbaren Resultate der Analyse:

$\begin{array}{ccc}\text { Angewandte Substanz: } & \text { Wasser: } & \text { Kohlensäure: } \\ \text { III. } 0,8675 & 0,2055 & 0,735 \\ \text { IV. } 3,7205 & 0,167 & 0,6095 \\ \text { V. } 0,7655 & 0,180 & 0,656\end{array}$

Die diesen Daten entsprechenden Kohlenstoffinengen würden 23,10 Proc., 23,07 und 23,37 sein; aber indem die oben besprochene Correction angebracht wird, so gehen sie resp. in 26,98 Proc., 26,95 und 27,25 über, welche den Zahlen, die die Formel erfordert, sehr nahe stehen, wie folgende vergleichende Tabelle ergiebt:

Berechnet:

\begin{tabular}{lrrccccc} 
& & I. & II. & III. & IV. & V. \\
$\mathrm{C}^{14}$ & 84,0 & 27,13 & - & - & 26,98 & 26,95 & 27,25 \\
$\mathrm{H}^{8}$ & 8,0 & 2,58 & - & - & 2,63 & 2,57 & 2,61 \\
$\mathrm{Ba}^{2}$ & 137,6 & 44,44 & 44,50 & 44,53 & - & - & - \\
$\mathrm{O}^{10}$ & 80,0 & 25,85 & - & - & - & - & - \\
\cline { 2 - 8 } & 309,6 & $100,00$. & & - & & &
\end{tabular}

Salicylsaurer Kalk. Man stellt dieses Salz mit derselben Leichtigkeit dar, wie das Barytsalz, indem man Salicylsäure mit überschüssiger Kalkmilch sieden lässt, wobei gewissermaassen die ganze Sänremenge in unlöslichen Niederschlage als neutrales Salz zurüickbleibt und die von dem freien Kalk mittelst der Kohlensäure befreite Lösung der Reaction der Salicylsäure beim Zusammenkommen mit Eisenchlorid nur in geringem Grade zeigt. Gleichwohl würde es schwierig sein, nach dieser Methode cin von kaustischem Kalk freies Salz darzustellen, und da andererseits das Kalkhydrat nur wenig in Wasser löslich ist, so würde man das Kalkwasser nicht anwenden können, um die Lösung des sauren Salzes zu präcipitiren, wie man behufs der Bereitung des Barytsalzes verfährt. Piria ist auf andere Weise dahin gelangt, diese Schwierigkeit zu überwinden, indem er eine Lösung von saurem salicylsaurem Kalk mit einer hinreichend concentrirten Lösung von Kalk in Rohrzucker niederschlug; aber diese Methode erfordert zum Gelingen einige unentbehrliche 
Vorsichtsmaassregeln. Eine concentrirte und mit Kalk gesïttigte Zuckerlösung trübt sich bekanntlich beim Erwärmen, indem sie eine basische Verbindung fallen lisst, deren Menge bei der Siedhitze dermaassen sich vermehrt, dass die Flüssigkeit zu einer weissen Masse von dem Aussehen des Stärkemehlwassers zum Steifmachen der Wäsche gerinnt. Da nun aber der salicylsaure Kalk in der Hitze bereitet wird, so erhellt, dass, wenn die angewandte Kalklösung einen Ueberschuss an Basis onthielte, die oben beschriebene unlösliche Verbindung sich bilden und mit dem salicylsauren Kalk vermengen würde. Darum muss die Lösung einen solchen Ueberschuss von Zucker enthalten, dass sie nicht die mindeste Trübung beim Erwärmen erleidet. Zu diesem Behufe bereitet man eine Lösung von Kalk in Zucker und prüft eine kleine Portion davon durch Frwärmen; trübt sich diese beim Erwïrmen, so setzt man mehr Zucker hinzu und prüft von Neuem, womit man $80^{\circ}$ lange fortfährt, bis bei der Temperatur der Siedhitze nicht die geringste Trübung mehr statt findet. Nachdem dies einerseits ausgeführt ist, bereitet man anderseits eine Lösung von saurem salicylsaurem Kalk, indem man Salicylsäure im Ueberschuss mit Wasser und kohlensaurem Kalk zum Sieden bringt und die siedende Lösung filtrirt. Um sich völlig gegen die gleichzeitige Bildung der basischen Verbindung von Kalk mit Zucker sicher zu stellen, löst man in der Flüssigkeit eine gewisse Menge sehr weissen Zuckers auf. Beide Lösungen werden für sich erhitzt und mit einander vermischt, wenn ihre Temperatur dem Siedepuncte sehr nahe ist, worauf man fortfährt, die Mischung ungefähr eine halbe Stunde im Marienbade zu erhitzen. Bei diesem Verfahren schlägt sich der salicylsaure Kalk als ein schwerer krystallinischer Sand in glänzenden Körnchen nieder.

Dieses Salz ist viel weniger löslich in Wasser, als der salicylsaure Baryt. Da es deshalb nicht möglich ist, dasselbe durch wiederholtes Uinkrystallisiren $\mathrm{zu}$ reinigen, so muss man sich begnügen, die Flüssigkeit abzufiltriren und den Niederschlag mit siedendem Wasser zu waschen. Obwohl sehr wenig löslich, hat der salicylsaure Kalk dennoch eine stark alkalische Reaction und wird durch Kollensäure zersetzt, wic das Barytsalz, weshalb man ihn unter einer Glocke neben kaustischem Kalk trocknen muss.

Das bei der Temperatur des siedenden Wassers dargestellte Kalksalz enthält 2 Aeq. Krystallwasser, welche 
es nur ïusserst langsam verliert, auch wenn man die Temperatur auf $180^{\circ}$ erhöht. Deshalb musste Piria, als er 1,4265 des Salzes bei stufenweise zunehmenden Temperaturen auf $180^{\circ}$ in einem Strome trockner und kohlensäurefreier Luft erhitat hatte, den Versuch mehrere Tage lang fortsetzen, ehe der sich offonbarende Gewichtsverlust eine bestimmte Grenze erreichte. Die Menge des Wasserverlustes betrug hierbei 0,1325 , was 9,29 Proc. entspricht. Die Formel $\mathrm{C}^{14} \mathrm{II}_{4} \mathrm{Ca}^{2} \mathrm{O}^{6}+2 \mathrm{Aq}$ würdo 9,26 Procent erfordern, eine mit der vorigen fast identische Zahl.

Um die Basis zu bestinmen, lat Piria cine gewogene Menge des Salzes mit concentrirter Schwefelsäure calcinirt und den Rückstand an der Iuft so lange geglüht, bis er völlig weiss wurde. Aus dem Gewichte des erhaltenen Sulfats wurde die Menge des Calciums berechnet. 0,4445 salicylsaurer Kalk lieferten 0,314 schwefelsauren Kalk. $0,797 j$ desselben Salzes, von einer andern 13ereitung herrülırend, linterliessen 0,5575 scliwefelsauren Kalk.

Dic Resultate der Fiementaranalyse differiren, wie es vorherzuschen war, hinsichtlich des Kohlenstoffgehalts von denen der Rechnung. Bei einem Versuche dieser Art lieferten 0,7505 salicylsauren Kalks 0,2115 Wasser und 1,0气̃ก̃ Kohlensäure.

Wenn man die aus den obigen Angaben abgeleiteten Zahlen mit den nach der Fommel $\mathrm{C}^{14} \mathrm{H}^{4} \mathrm{Ca}^{2} \mathrm{O}^{6}+2 \mathrm{Aq}$ bereclineten vergleicht, so hat man in 100 Theilen:

Berechnet:

\begin{tabular}{lrr}
$(14$ & 84 & 43,30 \\
$H^{6}$ & 6 & 3,09 \\
$C^{2} a^{2}$ & 40 & 21,62 \\
$O^{8}$ & 64 & \\
\hline & & 194.
\end{tabular}

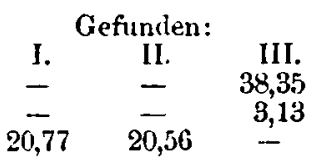

Der Versuch ergiebt also beinahe 5 Proc. Kohlenstoff zu wenig, während er hinsichtlich des Wasserstoffund Calciungrehalts der Rechnung entspricht. Wemn man annähme, dass aller Kalk in Verbindung mit Kohlensäure zurückbliebe, so müsste man dem erhaltenen Kohlenstoff $1 / 7$ der ganzen Kohlenstoffmenge des Salzes binzufügen, also $-\frac{43,30}{7}==6,19$, was eine Summe von 44,54 geben würde, sn dass die gcfundene Knhlenstoffimenge die der Formel um 1,24 Proc. überschreiten würde. Dieses Resultat erklärt sich aber ohne Schwierigkeit, wenn man 
bedenkt, dass bei der statthabenden Verbrennungstemperatur der organischen Substanzen der kohlensaure Kalk sich unvollständig zersetzt, indem er einen Theil seiner Kohlensäure entweichen lässt und einen Rückstand liefert, der ein Gemenge von kohlensaurem und kaustischem Kalk ist. Während also einerseits bei der Analyse der kalkhaltigen organischen Substanzen eine beträchtliche Menge von Kohlenstoff verloren geht, ist anderseits die bei den Barytsalzen benutzte Correction hier nicht anwendbar.

Bei dem Verfahren der organischen Elementaranalyse, welches Piria seit einigen Jahren in seinem Laboratorio anwendet, wird die organische Substanz in ein Metallschiffchen gelegt, welches man in dic Verbrennungsröhre hineinschiebt, wo es während der ganzen J)auer des Versuchs von dem Kupferoxyd entfernt bleibt. Diese Anordnung erlaubt, das Schiffchen nach Beendigung der Analyse aus der Röhre lervorzuzichen und den darin enthaltenen unorganischen Rückstand zu untersuchen. Auf eine sehr einfach Art, dic auf alle ähnliche fälle anwendbar ist, lässt sich die Menge der Kohlensäure experimentell bestimmen. Man schüttet die ganze Menge des Rückstandes, welche sich bequen aus dem Schiffehen entfernen lässt, in einen vorher abgewogenen bedeckten Platinticgel und wägt sie sehr schnell; darauf verwandelt man sie in schwefelsauren Kalk, indem man sie mit einem Ueberschuss von concentrirter Schwefelsäure calcinirt und wägt diesen. Aus diesen Daten lässt sich die mit dem Kalk verbundene Kohlensäuremenge berechnen. Bei Anwendung dieser Correction differiren die Resultate der Analyse nicht stärker von denen der Rechnung, als in den gewöhnlichen Fällen, wo die ganze durch die Verbrennung erzeugte Kohlensäuremenge in dem Kali-Apparat absorbirt wird.

0,387 salicylsaurer Kalk gaben bei der Verbrennung 0,1105 Wasser und 0,544 von der Kalilauge aufgenommene Kohlensïure. Andererseits hinterliessen 0,177 des kalkhaltigen Rückstandes dieser Analyse beim Calciniren mit Schwefelsäure 0,267 schwefels. Kalk. 0,267 schwefels. Kalk enthalten aber 0,110 Kalk; die ganze Menge des in 0,387 salicyls. Kalk enthaltenen Kalks betrïgt aber 0,112 , so dass im Ganzen 0,068 Kohlensäure zu der bei der Elementaranalyse gefundenen Menge zu addiren sind. Man erhält also $0,068+0,544=0,612$ Kohlensäure. In Folge dieser Correction enthalten 100 salicylsaurer Kalk nach der Analyse 


\section{Kohlenstoff . ... . 43,12 \\ Wasserstoff ..... 3,13}

Zahlen, welche sich von denen der Formel kaum unterscheiden.

Bleisalze der Salicylsäure. Cahours hat in seiner Arbeit über die Salicylsäure ein saures Bleisalz beschrieben, welches in spitzen, sehr glänzenden Nadeln krystallisirt, und das man erhält, wenn man Salicylsïure mit Wasser und kohlensaurem Bleioxyd sieden lässt und die Lösung siedendheiss filtrirt. Da Piria behufs der Darstellung der andern Bleisalze, wolche unten beschrieben werden, Cahours' saures Salz anwenden musste, so erhielt er mehrmals Gelegenheit, folgende Beobachtungen zu machen. Wenn man Salicylsäure, Wasser und kohlensaures Bleioxyd zusammen sieden lässt und letzteres Salz im Ueberschuss vorhanden ist, so besteht die Flüissigkeit, welche man erhält, so zu sagen nur aus reinem Wasser, oder kaum kann man eine Spur von aufgelöstem Blei entdecken, indem man die empfindlichsten Reagentien anwendet, wie z. B. Schwefelwasserstoff; und indem man sie mit Eisenchlorid versetzt, erhält man in sehr zweifelhafter Weise die Reaction der Salicylsäure. Das Resultat des Versuches ist ganz dasselbe, man mag das hydrathaltige kohlensaure J3leioxyd oder Bleiweiss des Handels, oder ein neutrales kohlensaures Bleioxyd anwenden, das man durch doppelte Wahlverwandtschaft erhält, indem man eine Lösung von essigsaurem Bleioxyd mit Soda niederschlägt. Man muss also, wenn man das saure salicylsaure Bleioxyd darstellen will, sich enthalten, einen grossen Ueberschuss von kohlensaurem Bleioxyd anzuwenden, und hätte man dies jemals gethan, so würde man der Flüssigkeit eine neue Menge Salicylsäure zusetzen müssen, so lange, bis der unlösliche Niederschlag sich völlig gelöst hätte. Wenn man dann siedendheiss filtrirt, so krystallisirt das saure Salz beim Abkühlen der Auflösung heraus.

Das kohlensaure Bleioxyd entzieht also die Salicylsäure einer wässerigen Lösung derselben, um eine in Wasser unlösliche oder beinahe unlösliche Verbindung zu bilden, welche folglich von dem von Cahours beschriebenen Salze verschieden ist. Der unlösliche Körper ist in ein schweres, weisses, krystallinisches Pulver verwandelt, welches alle Eigenschaften des neutralen salicylsauren Salzes besitzt, dessen Beschreibung unten folgt. Es würde sich also hieraus folgern lassen, dass das saure 
salicylsaure Bleioxyd das kohlensaure Bleioxyd zersetzen kann, um sich, indem es die Kohlensäure austreibt, in das neutrale Salz zu verwandeln. Nichts Analoges zeigt sich beim sauren Baryt- und Kalksalze, da sie beim noch so lange fortgesetzten Sieden mit den entsprechenden Carbonaten sich nicht nur nicht in neutrale Salze verwandeln, sondern da viclmehr die Kohlensäure den neutralen salicylsauren Baryt und Kalk zerlegt, indem sie einen Theil der Basis daraus niederschlägt und sie in die sauren Salze überführt. Das saure salicylsaure Bleioxyd besitzt übrigens eine Eigenschaft, welche diese anscheinende Anomalie crklärt: wenn man nämlich dieses Salz mit siedendem Wasser behandelt, so bleibt stets ein schweres, weisses, krystallinisches Pulver ungelöst zurück, welches auch bei wiederholter Behandlung mit neuen Mengen heissen Wassers sich nicht auflöst und ein neutrales salicylsaures Salz von der Formel $\mathrm{C}^{14} \mathrm{H}^{4} \mathrm{~Pb}^{2} \mathrm{O}^{2}$ ist. Dieses Verhalten zeigt, dass das saure salicylsaure Bleioxyd sich beim Sieden mit Wasser in das neutrale Salz und in freie Salicylsüure zerlegt. Wenn man nun annimmt, dass auch das kohlensaure Bleioxyd hinzutrete, so erhellt, dass die aus der Zersetzung des sauren salicylsauren Bleioxyds hervorgehende Salicylsäure auf das Carbonat einwirken wird, urn neucs saures Salz zu bilden, welches unter der Reaction der siedenden Flüssigkeit sich wiederum in Neutralsalz und freie Siuure zerlegen wird, und so fort, bis die ganze Menge der Salicylsäure in neutrales salicylsaures Bleioxyd verwandelt ist, welches sich absetzt.

Obwohl man das saure salicylsaure Bleioxyd in neutrales überfübren kann, indem man es einfach in Wasser sieden lässt, so ist doch das hierbei erhaltene Product in so wenig reichlicher Menge vorhanden, im Vergleich mit der angewandten Quantität des sauren Salzes, dass diese Methode für die Darstellung des Salzes nicht zu empfehlen sein würde. Man kann dagegen dasselbe leicht und in grosser Menge erhalten, indem man eine Auflösung von dreibasisch-essigsaurem Bleioxyd tropfenweise zu einer siedenden Lösung von saurem salicylsaurem Bleioxyd hinzusetzt. Das neutrale Salz schlägt sich als ein schweres, weisses, krystallinisches Pulver nieder, welches mit heissem Wasser abgewaschen und zwischen Löschpapier in einer durch Aetzkalk von Feuchtigkeit befreiten Atmosphäre getrocknet wird. Dieses Salz ist fast ganz unauflöslich in Wasser, besitzt alkalische Reactionen und wird durch freie Kohlensäure zersetzt. Beim Frwärmen bis 
1600 verliert es auch nicht eine Spur von Wasser. Seine Zusammensetzung entspricht der Formel $\mathrm{C}^{14} \mathrm{H}^{4} \mathrm{~Pb}^{2} \mathrm{O}^{6}$.

Ausser dem sauren und neutralen Bleisalze existirt noch eine andere Verbindung der Salicylsäure mit Bleioxyd, nämlich fünfbasisch-salicylsaures Bleioxyd. Man erhält dieses Salz leicht, indem man wenige Tropfen reines Ammoniak in eine siedende Lösung des sauren salicylsauren Bleioxyds giesst und die Mischung sieden lässt, bis der Anfungs entstandene Niederschlag sich in ein krystallinisches Pulver verwandelt hat. Nachdem dieses mit siedendem Wasser abgewaschen und dann unter einer Glocke neben Aetzkalk getrocknet worden ist, so hat es das Aussehen eines lejehten, weissen und krystallinischen Pulvers, welches unter dem Mikroskop geschen glimmerilhnliche Blättchen bildet. 1)ieses Salz, obgleich unlöslich in Wasser, besitzt eine genugsam wahrnehmbarc alkalische Reaction und verliert beim Erhitzen auf 1600 kein Krystallwasser. S'cine Zusammensetzung wird durch die Formel $\mathrm{C}_{14}^{14} \mathrm{H}^{4} \mathrm{~Pb}^{2} \mathrm{O}^{2}+3 \mathrm{PbO}$ dargestellt.

Kupfersalze der Salicylsäure. Fs existiren wenigstens zwoi Verbindungen von Kupferoxyd mit Salicylsiiure: ein saures und ein neutrales Salz. Das erstere, welches bisher noch von keinem Chemiker dargestellt worden war, besitzt etwas sonderbare Eigenschaften, und indem es sich unter verschiedenen Umständen zersetzt, verwandelt es sich in das neutrale Salz. Piria hat sich deswegen mit dem Studium dieser Verbindung beschäftigt, obwohl sie ausserhalb des Planes sciner Arbeit lag.

Saures salicylsaures Kupferoxyd. Man erhält es leicht durch doppelte Wahlverwandtschaft, indem man eine Lösung von schwefelsaurem Kupferoxyd und von saurem salicylsaurem Baryt vermischt und den Niederschlag von schwefelsaurem Baryt abfiltrirt. Wenn die angewandten Lösungen heiss und hinlïnglich concentrirt waren, so litsst die Flussigkeit beim Erkalten das saure salicylsaure Kupferoxyd so reichlich sich ausscheiden, dass sie völlig zu einer krystallinischen Masse gesteht, welche lange, spitze Nadeln von meerblauer, ins Grüne spielender Farbe bildet. Behufs der Reinigung muss man das Product zum zweiten Male krystallisiren lassen, nachdem man es von der Mutterlauge getrennt und zwischen Löschpapier getrocknet hat; da aber dieses Salz durch siedendes Wasser zersetzt wird, wie in der Folge gezeigt werden wird, so muss man beim Wiederaufösen desselben sorgfültig vermeiden, die Flüssigkeit höher als bis 
etwa 600 zu erwärmen. Die wässerige Auflösung des Salzes besitzt eine andere Farbe; diese ist ein schönes Grün, welches ins Gelbe spielt, ähnlich der Farbe des Kupferchlorïrs. Das Salz ist höchst löslich in Alkohol und Aether; aber letzterer übt eine höchst sonderbare zersetzende Einwirkung aus. Sobald nämlich der Aether mit dem Salze in Berührung kommt, so veründert sich die meerblaue Farbe desselben in Gelblichgriin, und gleichzeitig bildet sich eine intensiv grüne Auflösung. Wenn man eine grössere Menge Aether zusetzt und das Gemisch in einer geschmirgelten Flasche schüttelt, so erhält man eine grüne Lösung und ein unlösliches Pulver, welches wiederholt mit neuem Aether gewaschen, um jede Spur löslicher Materie abzuscheiden, das neutrale salicylsaure Kupferoxyd darstellt, dessen Beschreibung unten folgt. Das saure Salz verliert sämmtliches Krystallwasser bei einer Temperatur unter $100^{\circ}$, aber man kann nicht verhindern, dass mit dem Wasser zugleich Dämpfe von Salicvlsäure entweichen, welche sich in dem kälteren Theile dèr li'asröhre verdichten. Das entwässerte Salz ist dunkel olivenfarbig; über 1000 erwärmt, schmilat es und zerlegt sich gleichzeitig, indem es reichliche Dümpfe von Salicylsäure entwickelt. Im aufgelösten Zustande kann das saure salicylsaure Kupferoxyd bis zum Sieden erwärmt weyden, ohne sich zu zersetzen; wenn man aber das feste Salz in Beriihrung mit weniger Wasser, als zur Auflösung nöthig ist, erwärmt, oder wenn man scine wässerige Lösung so weit abdampft, dass eine Portion des Salzes sich am Boden des Gefässes auszusondern beginnt, so findet folgende Zersetzung statt. Das Salz verändert in beiden Fïllen ginzlich sein Ausseben, verliert seine krystallinische Beschaffenheit und verwandelt sich in eine harzartige, geschmolzene, dunkelgrüne Substanz, welche beim Erkalten vollkommen fest wird. Wenn man diese harzartige Materie mit einer neuen Menge heissen Wassers behandelt, so zertheilt sie sich, ohne sich zu lösen oder zu schmelzen, und verwandelt sich zuletzt in ein amorphes Pulver von grüner, ins Gelbe stechender Farbe, welches nichts anderes als das zweibasische salicylsaure Kupferoxyd ist.

Die bei dem so eben beschricbenen Processe angewandten Lösungen setzen beim Erkalten ein Gemenge von blauen Krystallen des sauren Salzes und von weissen Krystallen der Salicylsëure ab; bei hinreichender Verdünnung der Lösung krystallisirt nur Salicylsäure heraus.

Arch, d. Pharm. CXXXIV.Bds. 1.Hft. 
Aus diesen Thatsachen geht hervor, dass das saure salicyl. saure Kupferoyxd durch die Einwirkung der Hitze, des kalten Aethers, oder des heissen Wassers in zweibasisches Salz und Salicylsäure zerlegt wird, gerade wie die sauren schwefelsauren Salze der Alkalien unter denselben Bedingungen.

Die Analysen des sauren salicylsauren Kupferoxyds führen zu der Formel $\mathrm{C}^{14} \mathrm{H}^{5} \mathrm{Cu} \mathrm{O}^{6}+4 \mathrm{Aq}$.

Neutrales salicylsaures Kupferoxyd. Man kann es durch Zersetzung des sauren Salzes sowohl mitmeltst Aether, als mittelst siedenden Wassers darstellen, wie oben angegeben worden ist. Doch erhält man anf erstere Art nur kleine Quantititen, und bedarf einer grossen Menge Aethers. Ueberdies ist das Product niemals rein, und ausserdem, dass der Aether ihm hartnïckig anhingt, und nicht entfernt werden kann, ohne dass zugleich ein Theil des Krystallwassers fortgeht, hinterlässt es beim Verbrennen an der Luft eine Menge von Kupferoxyd, welche die durch die Formel vorgeschriebene um 1 oder 2 Proc. übersteigt. Dagegen ist das durch Zersetzung des sauren Salzes mit siedendem Wasser bereitete Product von constanter Zusammensetzung, der die Formol $\mathrm{C}^{14} \mathrm{H}^{4} \mathrm{Cu}^{2} \mathrm{O}^{6}+2 \mathrm{Aq}$ entspricht. Das salicylsaure Kupferoxyd ist ein leichtes, amorphes, gelblichgraues Pulver, welches hartnäckig den Gegenstïnden anhängt, mit welchen es in Berührung kommt; es ist unlöslich in Alkohol und Aether, ein wenig löslich in siedendem Wasser, dem es eine grüne Farbe mittheilt. Erwärmt verliert es sein Krystallwasser und nimmt eine braune Farbe an; gegen $180^{\circ}$ zersetzt es sich.

Salicylsaures Kupferoxyd-Kali. Dieses Doppelsalz ist unter den von $\mathrm{P}$ iria studirten das einzige in Wasser lösliche neutrale Salz der Salicylsäure. Man könnte wahrscheinlich verschiedene Methoden zu seiner Bereitung anwenden; aber Piria gebrauchte ausschliesslich folgende, welche sich eben so durch Leichtigkeit der Ausfiihrung, als durch Sicherheit des Resultats empfiehlt. Man löst zuerst weinsteinsaures Kupferoxyd in hinreichend concentrirter und in Ueberschuss vorhandener Kalilauge; dann löst man in der alkalischen Flüssigkeit krystallisirte Salicylsäure, indem man sie in kleinen Portionen auf einmal hinzufügt, und die Mischung fortwährend umrührt. Wenn unan so forttälurt, so wird in dem Maasse, als das Kali von der Säure gesättigt wird, die alkalische Flüssigkeit, welche vorher von rein azurblauer Farbe war, 
grïnlich; gleichzeitig beginnt ein krystallinisches, hellgrünes Pulver sich abzusetzen, und endlich gesteht die ganze Mischung zu einem Brei von Krystallen von gleicher Farbe, welche zum Trocknen auf einen Scherben von gebranntem, porösen Thon gelegt werden. Wenn man kein weinsteinsaures Kupferoxyd vorrïthig hat, so kann man statt dessen auch eine Lösung von schwefelsaurcm Kupferoxyd anwenden, zu welcher man Weinsteinsäure und Kalilauge im Ueberschuss zusetzt; aber die Operation gelingt weit besser nach der ersten Methode. Um das Product zu reinigen, muss man es ein zweites und selbst ein drittes Mal krystallisiren lassen, indem man $e s$ in der möglich kleinsten Menge warmen, aber nicht siedenden Wassers löst, weil es durch letzteres zerlegt werden würde. Das salicylsaure Kupferoxydkali krystal. lisirt langsam aus seiner wässerigen Auflösung in kleinen, rhombischen Tafeln, welche durchsichtig und von schöner, smaragdgrüner Farbe sind. Es ist sehr löslich in Wasser, aber unlöslich in Alkohol und Aether. Seine Lösung, welche intensiv grün gefärbt ist, wird durch Kalilauge nicht niedergeschlagen, selbst wenn letztere in grossem Ueberschuss ist; aber in letzterem Falle nimmt die Fltissigkeit eine dunkel azurblaue Farbe an, ähnlich der einer Lösung von wcinsteinsaurem Kupferoxyd in Kalilauge. W'enn man eine wässerige Lösung dieses Salzes sieden lässt, so zersetzt es sich, und schwarzes Kupferoxyd fällt nieder; zuletzt entfärbt sich die Flüssigkeit fast völlig, und lässt beim Abdampfen saures salicylsaures Kali auskrystallisiren. Das salicylsaure Kupferoxydkali verliert alles Krystallwasser, welches es enthält, wenn man es auf eine Temperatur bringt, die noch unter $100^{\circ}$ ist. Seine Zusammensetzung stellt die Formel $\mathrm{C}^{14} \mathrm{H}^{4} \mathrm{KCu} \mathrm{O}^{6}+$ $4 \mathrm{Aq}$ dar.

Salicylsaurer Kupferoxyd-Baryt. Die Darstellung dieses Salzes geschieht sehr leicht durch Zusatz von Chlorbarium zu einer Auflösung von salicylsaurem Kupferoxydkali : nach wenigen Augenblicken entsteht ein grüner, krystallinischer Niederschlag, dessen Farbe, obwohl minder intensiv, der des Scheeleschen Grün ăhnelt. Dies Salz ist fast unlöslich in Wasser. Erwärmt beginnt es bei $35^{\circ}$ Wasser abzugeben, und $100^{\circ}$ verliert es schon alles Krystallwasser. Die Analyse desselben ergiebt die Formel $\mathrm{C}^{14} \mathrm{H}^{2} \mathrm{BaCu} \mathrm{O}+4 \mathrm{Aq}$.

Aus diesen Thatsachen zieht Piria folgende Schlüsse:

1) Die Salicylsäure ist zweibasisch. 
2) Die alkoholisehen Derivate dieser Süure sind den Aethern der einbasischen Säuren nicht vergleichbar, sondern vielmehr wie die Weinsäuren einer vielbasischen Säure anzusehen, die folglich Methyl-, Aethyl-, AmylSalicylsäure zu nennen sind.

3) Die Beziehung zwischen der Sättigungscapacität der Salicylsăure und ihrer Devirate bildet keine Ausnabme, sondern ist dem allgemein für die Paarlinge geltenden Gesetze unterworfen. (Nuovo cimento, giornale di fisica, di chimica. Pisa, 1855. Januar, Februar.)

H. $B$.

\section{Veber einige neue wasserfreie Säuren.}

Wasserfreie Oenanthylsäure, ${ }_{\mathrm{C}^{14} \mathrm{H}^{13} \mathrm{O}^{2} !}^{\mathrm{C}^{13} \mathrm{O}^{2}} \mathrm{O}^{2}=\mathrm{C}^{28} \mathrm{H}^{26} \mathrm{O}^{6}$. Man erhält dieselbe nach $O$. Malerba, indem man 6 Aeq. vollkommen trocknes önanthylsaures Kali mit 1 Aeq. Phosphornxychloriir in Berührung bringt. Die Finwirkung erfolgt von selbst unter Wärmentwicklung, und kaum ist gegen das Ende des Processes cine Erwärmung nöthig. Die Darstellung wird auf gleiche Weise beendet, wie bei den übrigen wasserfreien Säuren.

Die wasserfreie Oenanthylsäure oder das önanthylsaure Oenanthyloxyd ist ein klares Oel vom spec. Gewicht 0,91 bei $14^{0}$. Sein Geruch ist in der Kälte schwach und zeigt einige Uebereinstimmung mit dem der wasserfreien Caprylsäure; beim Erwärmen wird er aromatisch. Lässt man die Sïure in schlecht verschlossenen Gefässen stehen, so nimmt sie einen ranzigen Geruch an. Die Alkalien zersetzen sie so wie alle wasserfreien Säuren, und Amoniakfliissigkeit verwandelt sie in Oenanthylamid.

Oenanthylsaures Benzoyloxyd, $\left.\mathrm{C}^{14} \mathrm{H}^{5} \mathrm{O}^{2}\right\}^{\mathrm{C}^{14}} \mathrm{O}^{13}=\mathrm{C}^{28} \mathrm{H}^{18} \mathrm{O}^{6}$. Man erbalt es leicht durch Einwirkung des Chlorbenzoyls auf önanthylsaures Kali. Es stellt ein Oel vom spec. Gewicht 1,043 dar. Sein Geruch ist dem der wasserfreien Oenanthylsäure ähnlich. Frisch dargestellt ist es neutral; an der feuchten Luft aber wird es sauer, und setzt Krystalle von Benzoësäurehydrat ab.

Oenanthylsaures Cumyloxyd, ${ }_{\mathrm{C}^{20} \mathrm{H}^{11} \mathrm{O}^{2}}^{\mathrm{C}^{14} \mathrm{H}^{13} \mathrm{O}^{2}} \mathrm{O}^{2}=\mathrm{C}^{34} \mathrm{H}^{24} \mathrm{O}^{6}$. Es wird durch denselben Process, wie die vorige Verbinbung, erhalten. Die Einwirkung geht loicht von Statten, und das Product ist ein Oel, welches leichter als Wasser 